\title{
Forschende Komplementärmedizin Research in Complementary Medicine
}

\section{Schriftleitung}

M. Bühring, Berlin

K. Linde, München

W. Marktl, Wien

D. Melchart, München

K. M. Meyer-Abich, Essen

K. L. Resch, Bad Elster

R. Saller, Zürich

M. Ullmann, München

\section{Wissenschaftliches Sekretariat}

Frau Dr. M. Ullmann

Frau I. Beer

Marienplatz 3

D-80331 München

Tel. +4989294770

Fax +4989294775

\section{Wissenschaftlicher Beirat}

S. Baumgartner, Bern Grundlagenforschung

A. Beck, Bern Neuraltherapie

R. Bloch, Bern Medizindidaktik

K.-M. Braumann, Hamburg Sport- und Bewegungsmedizin

F.-W. Dittmar, Starnberg Gynäkologie

G. Dobos, Essen Naturheilkunde und Integrative Medizin

F. Eitel, München Chirurgie und Evaluationsforschung

E. Ernst, Exeter Komplementärmedizin

A. Falkenbach, Badgastein-Böckstein Balneologie und Klimatologie

A. Gehrke, Hannover Physikalische und Rehabilitative Medizin

I. Gerhard, Heidelberg Gynäkologie

G. Glaeske, Bremen Evaluation medizinischer Versorgung

Chr. Gutenbrunner, Hannover Physikalische Medizin und Rehabilitation

F. Gutzwiller, Zürich Sozial- und Präventivmedizin

P. Heusser, Dornach und Bern Anthroposophische Medizin

G. Jendritzky, Freiburg i. Br. Medizin-Meteorologie

S. Jenny, Zürich Innere Medizin, Naturheilverfahren

W. B. Jonas, Bethesda Komplementärmedizin
H. Kiene, Freiburg i. Br. Wissenschaftstheorie

K. Kraft, Bonn Phytotherapie

K. W. Kratky, Wien Kybernetik und Wissenschaftstheorie

W. Kubelka, Wien Phytopharmakologie

G. T. Lewith, Southampton Komplementärmedizin

R. Lüdtke, Tübingen Statistik

R. W. März, Nürnberg Phytotherapie

P. F. Matthiessen, Herdecke Medizintheorie

B. Meier, Zürich Phytotherapie

H. Pietschmann, Wien Wissenschaftstheorie

R. Pothmann, Oberhausen Akupunktur

W. Schaffner, Basel Phytopharmazie

B. Schneider, Hannover Biometrie

A. Thurneysen, Bern Homöopathie

C. Uhlemann, Jena Physikalische und Rehabilitative Medizin

W. Vetter, Zürich Innere Medizin

H. Wagner, München Pharmazeutische Biologie

H. Walach, Freiburg i. Br. Psychologie 


\section{Bibliographische Dienste}

Index Medicus/MEDLINE

Current Contents ${ }^{\circledR} /$ Clinical Medicine

Science Citation Index Expanded

S. Karger $\cdot$ Medical and Scientific Publishers $\cdot$ Basel $\cdot$ Freiburg $\cdot$ Paris $\cdot$ London $\cdot$ New York $\cdot$ New Delhi $\cdot$ Bangkok $\cdot$ Singapore $\cdot$ Tokyo $\cdot$ Sydney

Die Zeitschrift erscheint zweimonatlich; pro Jahr erscheint 1 Band zu je 6 Heften. Bezugspreis für Jahrgang 7 , 1999: DEM 198,-/ CHF 154,- einschliesslich MwSt., zuzüglich Postgebühren. Der Abonnementpreis ist im voraus zahlbar. Das Abonnement der Zeitschrift läuft weiter wenn es nicht spätestens 4 Wochen vor Abschluss eines Bandes abbestellt wird. Abonnementbestellungen können bei jeder Buchhandlung oder direkt beim Verlag aufgegeben werden:

\section{Deutschland}

S. Karger GmbH

Lörracher Str. 16 a

D-79115 Freiburg

Tel. +49761452070

Fax +497614520714

E-mail Information@Karger.de Fax +41613061234

E-mail Karger@Karger.ch
Anzeigen:

S. Karger Verlag für Medizin und Naturwissenschaften $\mathrm{GmbH}$, Lörracher Str. 16a, D-79115 Freiburg, Tel +49 761452070. Gültig ist die Preisliste Nr. 3 vom 1. Januar 1999.

Für den Inhalt ausserhalb des redaktionellen Teiles (insbesondere Anzeigen, Industrieinformationen, Pressezitate und Kongressinformationen) übernehmen Schriftleitung, Beirat und Verlag keine Gewähr.

Eine Markenbezeichnung kann warenzeichenrechtlich geschützt sein, auch wenn bei ihrer Verwendung in dieser Zeitschrift das Zeichen ${ }^{\circledR}$ oder ein anderer Hinweis auf etwa bestehende Schutzrechte fehlen sollte. Für Satzfehler wa bestehende Schutzrechte fehlen sollte. Für Satzfehler,
insbesondere bei Dosierungsangaben, wird keine Gewähr übernommen.

Die Zeitschrift sowie alle in ihr enthaltenen einzelnen Beiträge und Abbildungen sind urheberrechtlich geschützt. Jede Verwertung, die nicht ausdrücklich vom Urheberrechtsgesetz zugelassen ist, bedarf der vorherigen Zustimmung des Verlags. Das gilt insbesondere für Vervielfältigung, Bearbeitungen, Übersetzungen, Mikroverfilmungen und die Einspeicherung und Verarbeitung in elektronischen Systemen.
(C) Copyright 2000 by S. Karger

Verlag für Medizin und Naturwissenschaften $\mathrm{GmbH}$ Lörracher Str. 16 a, D-79115 Freiburg

Verlagsleitung: Sibylle Hopf, Freiburg Presserechtlich verantwortlich: Marcela Ullmann, München

Redaktionsassistenz: Irmgard Beer, München Anzeigenleitung: Susanne Meister, Freiburg Produktionsleitung: Georg Brunner, Freiburg

Printed on acid-free paper by druckhaus köthen $\mathrm{GmbH}$ Friedrichstr. 11/12, D-06366 Köthen/Anhalt

\section{KARGER}




\section{Band 7, Heft 1, Februar 2000}

Editorial

4 Evidenz-basierte Komplementärmedizin - eine zukünftige Notwendigkeit

Melchart, D. (München)

Übersichtsarbeit

5 Mineralwasser oder Leitungswasser? Eine systematische Literaturanalyse zur Frage der mikriobiellen Sicherheit

Böhmer, H.; Resch, K.-L. (Bad Elster)

Originalarbeiten

12 Kirlian-Fotografie: Zufälliges oder personenspezifisches Entladungsmuster?

Treugut, H. (Schwäbisch Gmünd); Köppen, M.; Nickolay, B. (Berlin); Füß, R. (München); Schmid, P. (Schwäbisch Gmünd)

17 Teebaum-Öl: Eine systematische Übersicht randomisierter klinischer Studien

Ernst, E.; Huntley, A. (Exeter)

Gesellschaftsmitteilungen

21 SMGP Schweizerische Medizinische Gesellschaft für Phytotherapie

24 ESCNM European Society for Classical Natural Medicine

25 Karl und Veronica Carstens-Stiftung

26 Buchbesprechung

28 Industrieforum

Abstracts

29 Evidence-Based Complementary Medicine. State of the Evidence / Methodological Challenges

International Congress on Clinical Research and Quality Management in Complemetary Medicine, (EBCM-Kongress), München, 6.-8. April 2000

59 Tagungen und Kongresse

2 Impressum

61 Hinweise für Autoren

Peer Review: Jedes Manuskript wird von der Redaktion und von externen Gutachtern geprüft.
Vol. 7, Issue 7, February 2000

Editorial

4 Evidence-Based Complementary Medicine - a Need for the Future

Melchart, D. (München)

Review Article

5 Safety of Mineral Waters vs. Tap Waters. A Systematic Analysis of the Literature Concerning the Risk of Microbial Contamination

Böhmer, H.; Resch, K.-L. (Bad Elster)

Original Articles

12 Kirlian Photography: Accidental Pattern or Individual Specific Pattern?

Treugut, H. (Schwäbisch Gmünd); Köppen, M.; Nickolay, B. (Berlin); Füß, R. (München); Schmid, P. (Schwäbisch Gmünd)

17 Tea Tree Oil: A Systematic Review of Randomized Clinical Trials

Ernst, E.; Huntley, A. (Exeter)

Society Bulletins

21 SMGP Schweizerische Medizinische Gesellschaft für Phytotherapie

24 ESCNM European Society for Classical Natural Medicine

25 Karl und Veronica Carstens-Stiftung

26 Book Review

28 Industrial Forum

Abstracts

29 Evidence-Based Complementary Medicine. State of the Evidence / Methodological Challenges

International Congress on Clinical Research and Quality Management in Complemetary Medicine, (EBCM-Kongress), Munich, April 6 to 8, 2000

59 Meetings and Conferences

2 Imprint

63 Guidelines for Authors

Peer Review: Each manuscript is evaluated by the editors and by externa peer reviewers.

\section{KARGER}

Fax +497614520714

E-mail Information@Karger.de

www.karger.com 